\title{
Fecundidad diferencial de distintas subpoblaciones de mujeres en España: una diferencia no tan grande
}

\author{
Graciela Sarrible \\ Universitat de Barcelona \\ gsarrible@yahoo.com
}

Recibido: 08-12-2008

Aceptado: 15-03-2009

\section{Resumen}

La sombra de las grandes diferencias de fecundidad se cierne otra vez sobre las poblaciones europeas. La aportación al crecimiento natural de las poblaciones foráneas constituyó un Leitmotiv en demografía en el siglo pasado. El miedo no se basa en el tamaño de la familia, es decir, en la cantidad de hijos que tienen las mujeres forasteras, sino en las consecuencias que ello puede conllevar a largo plazo. La cuestión que provoca temores se centra en la proporción en que las generaciones futuras estarán formadas por personas cuyos padres serán de fuera. La principal diferencia que se ha observado hasta el presente reside en los tiempos en que se tienen los hijos, y no exclusivamente en el resultado final. El objetivo de este trabajo consiste en contrastar las diferencias de natalidad y fecundidad entre el conjunto de España y las mujeres extranjeras que viven en el país. La hipótesis que se presenta a comprobación enuncia que la distancia cuantitativa entre los indicadores de fecundidad de ambos grupos no es tan grande como se supone $\mathrm{u}$ otros indicadores brutos hacen pensar. Se impone una discusión metodológica sobre el tema, puesto que cada índice representa un aspecto diferente de la fecundidad de las mujeres.

Palabras clave: fecundidad diferencial, España, mujeres extranjeras.

\begin{abstract}
Fertility of different segments of women in Spain: a not so large gap
The shadow of a large gap in fertility looms again over the European populations. During the last century, the contribution of the foreign population to natural population growth constituted a leitmotiv in Demographics and other disciplines. The concern thereby is not based on the number of members of a family; that is the quantity of children that foreign women have, but on the long-term consequences of this matter. The question that provokes anxiety is centered on the proportion of foreign parents or parents with foreign origins of future generations. The principal difference that has been observed until now comprises the moment in which women have their children, and not exclusively the final number of children. The aim of this paper is to contrast the differences of fertility and birth rates between the Spanish female population and foreign women who currently live in the country. The hypothesis which will be demonstrated formulates that the gap between different indicators of fertility is not as big as supposed and as other raw indicators made us think. A methodological discussion is imposed about this issue because each indicator represents a different aspect of fertility of women.
\end{abstract}

Key words: differential fertility, Spain, foreign women. 


\section{Sumario}

1. Indicadores usuales: natalidad y fecundidad

2. Modelización
3. Conclusión: diferencias de fecundidad presentes y menores futuras

Referencias bibliográficas

La sombra de las grandes diferencias de fecundidad se cierne otra vez sobre las poblaciones europeas. Siempre se ha temido al de fuera. La aportación al crecimiento natural de las poblaciones foráneas constituyó un Leitmotiv en demografía en el siglo pasado. El miedo no se basa en el tamaño de la familia, es decir, en la cantidad de hijos que tienen las mujeres forasteras, sino en las consecuencias que ello puede conllevar a largo plazo. La cuestión que provoca temores se centra en la proporción en que las generaciones futuras estarán formadas por personas cuyos padres serán emigrantes.

Referente a la fecundidad diferencial, es decir, la cantidad de hijos que tienen las mujeres de uno u otro grupo, ha habido objetores y detractores. Incluso las conclusiones de las investigaciones parecerían contradictorias si no prestásemos atención a las cuestiones metodológicas que las diferencian (Andorka, 1982). Un estudio de la fecundidad de las mujeres de nacionalidad extranjera en comparación con las del conjunto de España daría como resultado la cercanía entre los indicadores de fecundidad de los distintos grupos. La diferencia principal que se ha observado hasta el presente reside en los tiempos en que se tienen a los hijos y no en el resultado final (Sarrible, 1986, 2005 y 2008).

El objetivo de este trabajo consiste en medir las diferencias de fecundidad entre el conjunto de España y las mujeres extranjeras que viven en el país. La hipótesis que se presenta a comprobación enuncia que la distancia cuantitativa entre los indicadores de fecundidad de ambos grupos no es tan grande como se supone u otros indicadores brutos hacen pensar. Se especula mucho sobre el aporte al crecimiento natural, pero poco se ha cuantificado acerca de las verdaderas diferencias entre las poblaciones (Sarrible, 2004 y 2008).

La literatura sobre fecundidad ha pasado décadas sentenciando que las cifras de descendencia de unas era elevada y las de otras, insuficiente, salvo algunos autores que se han mofado de esta contradicción (Andorka, 1982). Todo depende de la medida en que esa fecundidad se aleja del crecimiento cero de la población, que, por ahora, sigue constituyendo un mito no alcanzado. Si bien no me adhiero a la teoría de la transición, que con muchos otros estimo superada, la propuesta de Bongaarts y Cotts Watkins (1996) de la interacción social me seduce. En realidad, resulta muy difícil explicar por qué motivo las poblaciones que viven juntas se asemejan, pero esa noción podría ser aplicada al futuro de los que habitan hoy y más tarde habitarán España.

Los indicadores que aquí se presentan se refieren todos a la natalidad y a la fecundidad. Por necesidad, también deben analizarse los denominadores que han servido, por lo que se hace una referencia necesaria a la estructura del 
conjunto de mujeres extranjeras en edad fecunda que viven en España. A pesar de que, en la actualidad, sólo existiría una cantidad de referencia (el padrón), hace algunos años las diferencias entre los guarismos que cuantificaban a estas poblaciones podían ser extremadamente elevadas (Sarrible, 200.

Durkheim (1895) sentenció hace ya mucho tiempo, prácticamente durante el nacimiento de la sociología empírica, que «los datos no son los hechos». Frase que permite distinguir y recordar que las cifras intentan recoger, de la mejor manera posible, hechos como los nacimientos o las migraciones, pero que no tienen carácter exhaustivo, sino aproximativo.

La comparación se establece entre el total de España y las mujeres extranjeras. Se ha considerado pertinente no calcular un colectivo de mujeres de nacionalidad española, sino tener de referente a la cifra que las incluye a todas. En la primera parte, se cuentan los indicadores usuales de natalidad y fecundidad. En la segunda parte, se construye un modelo para imaginar una situación ficticia donde las extranjeras muestran la fecundidad de las españolas o tienen la estructura de edad de las españolas, para poder distinguir las dos principales razones de la diferencia entre ambas poblaciones. En esta segunda parte, se incluye una modelización por continente y se compara con la situación real.

A fin de poder establecer la diferencia entre las poblaciones, se comienza por la evolución de los nacimientos. En segundo lugar, se calculan las tasas de natalidad. La referencia a la proporción de nacimientos entre mujeres muy jóvenes resulta obligada, puesto que, normalmente, se considera un indicador de fecundidades elevadas, llamadas pretransicionales. En último lugar, se calcula la tasa de fecundidad global, puesto que servirá para realizar la modelización posterior.

En la segunda parte, se realizarán estimaciones de situaciones ficticias para poder distinguir el peso absoluto de los niveles de fecundidad de cada colectivo, con independencia de las estructuras de población. Esto significa que se pueden establecer comparaciones entre ambas poblaciones, como si las poblaciones tuvieran la misma estructura de edades. Se calcula un segundo modelo, complementario del anterior, donde se mantendrán invariables los niveles de fecundidad y ambas poblaciones se diferenciarán por su estructura. Dado que los datos de nacimientos no nos permiten realizar el primer modelo desagregado por continente, se hace sólo el segundo. Se dispone de las distribuciones de las mujeres extranjeras en edad fecunda por continente.

Los datos provienen de las estadísticas oficiales del INE. Estudios anteriores (Sarrible, 2004, 2005, 2006 y 2008) han planteado tipos variados de errores y de problemas en el tratamiento de estos temas, puesto que puede haber dificultades con los denominadores. Los datos no están desagregados como para realizar más cálculos. En esa medida, los estudios basados en datos publicados resultan limitados, con lo que solamente pueden constituir una primera y necesaria aproximación a este tema. Lo primero que se debe hacer es cuantificar e intentar dilucidar las razones, aunque estudios más profundos, con otros medios, pueden llegar más lejos en su búsqueda. 


\section{Indicadores usuales: natalidad y fecundidad}

Los nacimientos están aumentando en España desde hace muchos años, y ello no sólo se debe al aporte extranjero, sino también a un incremento de la fecundidad por parte de las mujeres españolas. Ello debería ser motivo de alegría para aquellos agoreros que se quejaban de los niveles bajos de las décadas anteriores y que los llevaron a realizar discursos negros, en los cuales reclamaban abiertamente que las mujeres tuvieran hijos. En el presente artículo, se va a analizar la evolución de los nacimientos desde 1998 hasta el último dato publicado: 2006.

En la tabla 1, la evolución del primer índice basado en el total de nacimientos habidos en España en dicho período muestra que el crecimiento en estos años ha sido prácticamente de un tercio respecto a la cantidad inicial. Sin embargo, el total de hijos de madres extranjeras ha crecido más de cinco veces en el mismo período.

Los números índices reflejan la diferencia en el ritmo de crecimiento entre el total y el colectivo de mujeres extranjeras. Al mismo tiempo que la cantidad de hijos de madres extranjeras crecía, aumentaba su peso en el total de nacimientos, pero la proporción se ha incrementado sólo 2,5 veces. En realidad, este ritmo parece, a simple vista, mucho más lento que el calculado en una ocasión anterior (Sarrible, 2008).

En la primera presentación de los indicadores, en que el período comenzaba en 1998, los partos de las madres extranjeras se multiplicaban por cinco hasta 2006 y la proporción respecto al total aumentaba cuatro veces. Si dejamos de lado, como se ha hecho en este caso, los tres primeros años, el ritmo de crecimiento resulta más suave para las madres extranjeras y se ven pocos cambios para el total.

De esta manera, lo que puede parecer alarmista en un estudio (que los nacimientos se incrementen cinco veces y la proporción, cuatro), puede cambiar

Tabla 1. Evolución de los nacimientos en España en el siglo XXI (2001-2008). Total de España y datos correspondientes a madres extranjeras. Índices y proporción.

\begin{tabular}{llcc}
\hline & \multicolumn{2}{c}{ Índices } & \\
\cline { 2 - 3 } Año & Total & Extranjeras & Proporción* \\
\hline 2001 & 100 & 100 & 8,24 \\
2002 & 103 & 132 & 10,55 \\
2003 & 109 & 161 & 12,7 \\
2004 & 112 & 187 & 13,78 \\
2005 & 115 & 210 & 15,07 \\
2006 & 118 & 236 & 16,46 \\
2007 & 121 & 279 & 18,98 \\
2008 & 128 & 321 & 20,71 \\
\hline
\end{tabular}

Sobre la fuente: Los datos del año 2008 son todavía provisionales. La última consulta en el INE se realizó en febrero de 2010.

*La proporción se encuentra dividiendo los nacimientos de madres extranjeras por el total. Elaboración propia. 
sólo alternando el año de referencia y comparación. Dejando de lado, como en este caso, dichos primeros años, el ritmo de crecimiento aparece más sosegado: tres veces en lugar de cinco para las madres extranjeras, y su aportación al total, si bien crece igual y continúa creciendo en los últimos años, no lo hace al mismo ritmo que si tomásemos un año inicial de baja aportación.

¿Cuáles son los posibles factores que pueden provocar este incremento? Al menos, se pueden mencionar tres:

- En primer lugar, el aumento de la población extranjera.

- En segundo lugar, y posible pero no comprobable directamente con estos datos, el deseo de completar o aumentar la familia después de un lapso de tiempo en el que se ha migrado y se ha postergado algún nacimiento. Esta es una de las propuestas de Andorka (1982) para considerar la influencia de la migración en la fecundidad.

- En tercer lugar, una posible fecundidad diferencial, la cual es motivo de reflexión en este trabajo, igual como lo ha sido en múltiples investigaciones (De Valk et al., 2004).

El primer factor se puede cuantificar. A continuación, se calculan las tasas de natalidad que tienen en cuenta como denominadores a la población extranjera. El segundo factor ha sido apuntado en otros trabajos como explicación posible, pero no se puede demostrar con los datos disponibles (Sarrible, 2004). El tercer factor forma parte casi de los mitos que establecen diferencias entre las poblaciones. Cabe decir que hay que estudiar cada caso para poder establecer la veracidad o la falsedad de la afirmación (Sarrible, 1986).

La proporción de la población extranjera en el total de los habitantes de España ha pasado de un 1,53 \% en el año 1998 a un 6,24 \% en el 2003 y a un $10 \%$ en el 2007 , y, de acuerdo con los datos provisionales del padrón del 1 de enero de 2009, la proporción alcanzaría el 12,08 \% (INE, 2008-2010). Estas cifras muestran un incremento rápido al inicio y más lento, pero permanente, en los años siguientes. Por sexos, las proporciones no son idénticas, aunque sí muy próximas. Hay mayor cantidad de hombres, puesto que representan el $10,72 \%$ de la población masculina del país frente al 9,29\% de las mujeres extranjeras respecto al total correspondiente al año 2007. Las diferencias debidas a la edad de la población merecen ser destacadas, aunque son las normales entre la de unos individuos migrantes — que son más jóvenes cuando llegan al nuevo país - y la de los habitantes locales.

En la tabla 2, de elaboración propia, constan las tasas de natalidad calculadas por el INE para el total de España y las correspondientes a las madres extranjeras. Evidentemente, esta es una de las series históricas clave para entender el crecimiento natural de la población española. Las nuevas estimaciones de la población española del INE han aumentado los denominadores, lo que implica que el fenómeno pudo haber sido sobreestimado en cálculos realizados en años anteriores, al considerar que la población de referencia era menor. Lo mismo podría ocurrir con la población extranjera, que constituye el denominador de la segunda tasa. 
Tabla 2. Tasas de natalidad. Total de España y de madres extranjeras con números índices correspondientes al período 2000-2008

\begin{tabular}{lccc}
\hline Años & Tot. Esp. & Extranj. & Índice \\
\hline 2000 & 9,88 & 23,22 & 2,35 \\
2001 & 9,98 & 20,03 & 2,01 \\
2002 & 10,14 & 19,06 & 1,88 \\
2003 & 10,52 & 18,96 & 1,89 \\
2004 & 10,65 & 18,62 & 1,75 \\
2005 & 10,75 & 17,84 & 1,66 \\
2006 & 10,92 & 18,26 & 1,67 \\
2007 & 10,98 & 19,11 & 1,74 \\
2008 & 11,38 & 19,69 & 1,73 \\
\hline
\end{tabular}

Sobre los indicadores:

1. Las tasas de natalidad del total de España son del INE. Las consultas se realizaron en enero de 2008 y en febrero de 2010.

2. Las tasas están expresadas en tantos por mil.

3. El índice es el resultado de la división entre la tasa de extranjeras y la del total de España.

4. Sobre las fuentes: Los datos de 2008 todavía son provisionales.

Las tasas de natalidad del conjunto de la población extranjera que vive en España han sido calculadas de acuerdo con los datos de los padrones. En estos momentos, se ha unificado el criterio y sólo hay una población de referencia para españoles y extranjeros, lo que facilita enormemente el trabajo, puesto que, a diferencia de antes, deja de haber alternativas en que las poblaciones de referencia podrían ser múltiples.

La última columna de la tabla 2 corresponde al número índice que relaciona las tasas anteriores del total de España y de las poblaciones extranjeras. En este cálculo, se divide la mayor cifra de natalidad por la menor, o sea, la tasa de extranjeras respecto de la del total de España.

Mientras que la serie de tasas de natalidad del total de España es creciente sin excepción, la correspondiente a la población extranjera presenta oscilaciones. Va en sentido inverso hasta el año 2005 y luego vuelve a crecer, por lo cual es lógico que se acerquen ambas tasas. De una diferencia superior al doble, pasa a ser menor conforme avanzamos hacia el presente. Se puede decir, entonces, que las diferencias medidas por la tasa de natalidad entre el total de España y la población extranjera disminuyen de manera constante.

Una de las distinciones más salientes entre las poblaciones local y extranjera se refiere a la edad de iniciarse en la maternidad y la frecuencia con que se tienen hijos cuando se es muy joven. Las mujeres de menos de veinte años representan a un grupo de referencia y de riesgo, por ello las políticas sanitarias van encaminadas a reducir los embarazos en adolescentes. Un embarazo temprano ahonda la desigualdad de género, en la medida en que ellas se ven afectadas por la maternidad, interrumpen su educación y limitan sus posibilidades de formación futura y mejora laboral, mientras que los hombres rara vez alteran su vida por esta razón. Por ello, los embarazos de mujeres jóvenes son estudiados y tratados aparte (Sarrible, 2006). 
Tabla 3. Tasas de fecundidad global del total de España y de madres extranjeras (período 2000-2008). Tasas y número índice

\begin{tabular}{lrrc}
\hline Años & Tot. Esp. & Extranj. & Índice \\
\hline 2000 & 38,1 & 74,74 & 1,96 \\
2001 & 38,53 & 62,44 & 1,62 \\
2002 & 39,19 & 59 & 1,51 \\
2003 & 40,76 & 58,25 & 1,43 \\
2004 & 41,39 & 56,88 & 1,37 \\
2005 & 41,95 & 55,13 & 1,31 \\
2006 & 43 & 56,25 & 1,31 \\
2007 & 43,3 & 58,68 & 1,36 \\
2008 & 45,15 & 60,98 & 1,35 \\
\hline
\end{tabular}

Sobre los indicadores:

1. Las tasas de natalidad del total de España son del INE. Las consultas se realizaron en enero de 2008 y en febrero de 2010.

2. Las tasas están expresadas en tantos por mil.

3. El índice es el resultado de la división entre la tasa de extranjeras y la del total de España. Sobre las fuentes:

4. Los datos de 2008 todavía son provisionales.

La fecundidad más temprana en las mujeres venidas de fuera resulta un enunciado arquetípico de un modelo con una intensidad más elevada. Sin embargo, sería deseable, según las políticas enunciadas para estos grupos, que pudieran retrasar la maternidad, tal como se ha mencionado anteriormente.

Con las tasas de fecundidad global ocurre lo mismo que con las de natalidad: van en sentido inverso (tabla 3). Mientras que las del total de España resultan crecientes, las que corresponden a las extranjeras son decrecientes. Ello provoca el mismo efecto que en el caso de la natalidad: se acercan. Si continúa esta tendencia, las diferencias serán todavía menores con los años. Si al inicio no llegaban al doble, al final no alcanzan un tercio.

Las diferencias entre las tasas de fecundidad global pueden ser menores que las de natalidad, porque los datos estudiados sólo tienen en cuenta a las mujeres en edad fecunda y no a la población en general. La población española está mucho más envejecida que las personas que han arribado estos últimos años a vivir en nuestro país, por ello, dentro de unos años, también convergerán las pirámides. De momento, las diferencias todavía son sustanciales.

Como se ha mencionado anteriormente, el cálculo de poblaciones tiene mucho que ver con la dimensión de las tasas. Las series de estimaciones previas de la población de mujeres españolas llegan al año 2004 inclusive. En cambio, a partir de 2005, se realizan nuevas estimaciones. En esa medida, no se debe interpretar como un verdadero descenso, sino como un cálculo más ajustado a la realidad. La proporción de mujeres extranjeras en edad fecunda no alcanza a ser tres veces superior en el período, pero resulta próxima. Es una buena razón para que aumenten los nacimientos en el total.

De acuerdo con estudios anteriores (Sarrible, 2004, 2005), los colectivos de extranjeros no se inscriben de la misma manera ni en la misma proporción en 
Tabla 4. Poblaciones medias de mujeres utilizadas en los cálculos de las tasas globales. Proporción de las extranjeras en el total de España en edad fecunda del 1 de julio de 2001 al 2008

\begin{tabular}{lc}
\hline Año & Proporción \\
\hline 2001 & 5,88 \\
2002 & 8,21 \\
2003 & 10,19 \\
2004 & 11,58 \\
2005 & 11,18 \\
2006 & 12,25 \\
2007 & 13,72 \\
2008 & 15,4
\end{tabular}

Sobre las fuentes: Los datos sobre las poblaciones del total de España y de las madres extranjeras han sido obtenidos del INE.

Elaboración propia.

que están presentes en España en los registros tales como el padrón. Ello implica que los denominadores pueden no contener a toda la población, aunque el nivel de error no es el mismo, puesto que tiene que ver con el lugar de origen de los emigrantes. Es incorrecto suponer que, en conjunto, actúan de la misma manera y que las equivocaciones o subdeclaraciones afectan a todos por igual.

Las personas que provienen de América han demostrado una tendencia mayor a inscribirse que las personas de cualquier otro origen continental. Eso puede redundar en mayores probabilidades de obtener la residencia cuando se conceden amnistías (Sarrible, 2004).

Por ello, las tasas globales de fecundidad expresan mucho mejor la proximidad, pero, sobre todo, el acercamiento entre las tendencias de ambos grupos. $\mathrm{Al}$ igual que en el caso de la natalidad, las tasas globales de fecundidad del total de España se incrementan, mientras que las tasas de las madres extranjeras presentan oscilaciones, primero a la baja y, en los últimos años, al alza. Aquí se puede recordar la explicación de Andorka (1982), que proponía una recuperación una vez finalizado el período de adaptación, aunque este extremo resulta difícil de probar fehacientemente si nos basamos en los datos disponibles. En la misma medida, la diferencia inicial, que era casi del doble, se ve reducida a prácticamente un poco más de un tercio al final. En los últimos años, ha oscilado en torno a esa diferencia. Otro punto en común es el incremento de ambas tasas a continuación del mínimo correspondiente a 2005.

Cuanto más precisamos a las poblaciones de referencia, más próximos resultan los indicadores. Cuanto más exactas son las cifras, más se puede considerar lo que sucede y menos estamos a merced de estimaciones que se alejen de la realidad. Al pasar de la población total, que es el denominador de la tasa de natalidad, a la población de mujeres en edad fecunda, que lo es de la tasa de fecundidad global, los resultados se aproximan. Al mejorar las estimaciones de las mujeres españolas en edad fecunda, los indicadores sufren algunas alteraciones que no deben ser explicadas por otro argumento que una mejora de las cifras. 
Pero no basta con esa precisión. La distribución de las mujeres en edad fecunda, pero más aún la frecuencia de los nacimientos a las distintas edades, también es diferente. La maternidad temprana de las adolescentes resulta un reto para el futuro, ya que las distancias han crecido en vez de disminuir. La proporción de nacimientos entre madres adolescentes y extranjeras alcanza el doble en algún momento de este período (Sarrible, 2006 y 2008). Los partos de madres extranjeras de menos de veinte años cada vez representan una proporción mayor del total de dicha edad para el conjunto del país. Y ese es el verdadero problema: que sigan creciendo mientras que otras proporciones o diferencias menguan.

$\mathrm{Si}$, en 1998, los nacimientos correspondientes a madres extranjeras respecto al total de mujeres de la misma edad (menores de veinte años) representaban el 6,25\% del total; en el 2000 ya son uno de cada diez; en el 2003, un poco más de uno de cada cuatro, y en el 2007, la proporción alcanza el $36,86 \%$. El incremento ha sido continuo y sostenido, y no se observa ninguna inflexión o cambio, como en los demás casos.

\section{Modelización}

En este apartado, se propone una simulación, es decir, se consideran situaciones ficticias donde uno de los dos factores que influye en la procreación es anulado. Nos referimos a la estructura de edades de las mujeres fértiles, que puede ser favorable o desfavorable a la fecundidad, de acuerdo con su distribución y los propios niveles de fertilidad. $\mathrm{Al}$ anular un factor, el otro puede ilustrar las diferencias entre el total de España y el de las mujeres extranjeras.

Para ello, primero se han calculado, a partir de los datos del INE sobre nacimientos y mujeres empadronadas, las tasas de fecundidad por grupo de edad para las mujeres extranjeras. De los cálculos realizados, se deduce que la procreación del total de España resulta relativamente tardía, en cambio, la de las extranjeras es más temprana, puesto que la tasa mayor tiene lugar diez años antes y las más elevadas se sitúan antes de los treinta.

El índice sintético de fecundidad (ISF), indicador transversal que se puede calcular de estas tasas, se mide como el número medio de hijos por mujer. Esta medida estrictamente transversal no es aplicable a largo plazo. En este caso, se ha calculado para poder establecer una comparación entre ambas poblaciones. Mientras que el número medio para el total de España alcanza 1,35 hijos, el de las extranjeras es de 1,73. De acuerdo con este indicador sintético que tiene en cuenta la fecundidad por edad, la diferencia en el año 2005 sería de un $28 \%$ entre ambas poblaciones de mujeres, lo que significa cada vez menor distancia entre las dos.

\section{Modelo de estandarización de tasas}

La técnica de estandarización de tasas sirve para poder establecer comparaciones entre poblaciones que se estiman diferentes sobre todo debido a la distri- 
bución por edades, como es el caso y ya ha sido expuesto. Además, suple la carencia de algunos datos por categorías. Sirve, de esta manera, para superar las comparaciones más simples, al eliminar el denominado efecto estructura.

Se trata de estandarizar las tasas de fecundidad por grupos de edad de cada población. Se utiliza una sola estructura para ambas poblaciones y las tasas de fecundidad correspondientes a cada una de ellas. El resultado representa exactamente los niveles de fertilidad de cada población, en ausencia del efecto estructura. En la medida en que se usa una estructura común, no hay distorsión alguna, puesto que en todos los casos es la misma. De esa manera, el resultado refleja, exclusivamente, la diferencia debida a los niveles de fecundidad, si la estructura de edades fuese la misma (que no lo es).

La tasa de fecundad global del total de España para 2005 es de un 41,95 por mil. La misma tasa para las extranjeras sin efecto estructura sería de un 50,05 por mil. Las diferencias entre las tasas de fecundidad global del total de España y de las extranjeras, para 2005, serían:

- Con efecto estructura: un $31,4 \%$.

- Sin efecto estructura: un 19,3\%.

Eso significa que ese $31,4 \%$ de diferencia entre el total de España y las mujeres extranjeras, que es una diferencia real, obedece a ambos efectos: la distribución por edades de las distintas subpoblaciones y los diferentes niveles de fecundidad. Con este cálculo, se ha eliminado el primer factor, por lo que se puede afirmar que la diferencia entre los niveles de fecundidad del total de España y del conjunto de mujeres extranjeras sería menor de un $20 \%$, si ese fuera el único motivo de diferencia entre ambos colectivos. La proporción restante corresponde a la estructura de edades más favorable entre las extranjeras. Cabe decir que este modelo ha permitido distinguir entre ambos factores.

\section{Conclusión: diferencias de fecundidad presentes y menores futuras}

Es absurdo buscar la igualdad entre grupos. Jamás la ha habido, ni en materia de fecundidad, ni en otras cuestiones demográficas o culturales. El mito de la integración entendida como homogeneización ha sido abandonado, no se sabe si por absurdo o por imposible.

Las diferencias entre ambas poblaciones (total de España y mujeres extranjeras) no son tan importantes como algunos pretenden hacernos creer. Se han aproximado de manera notable durante largos años, aunque, en los últimos tiempos, un nuevo incremento en las poblaciones extranjeras ha impedido que continuara esa aproximación. A pesar de ello, también deben ser tenidos en cuenta otros factores (Sarrible, 2004).

En un extremo, están los números absolutos de nacimientos, con su contribución al crecimiento natural del año. Las tasas de natalidad representan la forma de referir esos nacimientos a las poblaciones que han concebido a esos niños: hombres y mujeres sin límite de edad. En el año 2000, la diferencia entre una tasa y otra era del simple al doble. En el año 2005, se reducía a dos 
tercios, pero con posterioridad ha vuelto a acrecentarse. Sin embargo, en ningún caso hay un retorno a las diferencias iniciales.

Si se estudia un grupo específico, como las adolescentes o menores de veinte años, se pueden considerar dos aspectos. Si bien es cierto que la proporción de hijos a esa edad entre las extranjeras duplica a la que se refiere al conjunto de España, lo grave es que cada vez contribuyen con más nacimientos, puesto que han pasado a representar más de un tercio de los partos a esas edades en el año 2007. Si más de uno de cada tres hijos a esa edad es de madre extranjera, se deberían aplicar una política y un plan de salud específicos que deberían tener en cuenta a las mujeres que pueden padecer riesgo de embarazo.

Las diferencias entre las tasas globales de fecundidad que reducen el denominador a la población de mujeres en edad fecunda muestran que eran de prácticamente el doble al inicio, pero un poco más de un tercio las separa al final. Cuanto más específico es un indicador, excepto el caso de la fecundidad temprana, más se reducen las diferencias.

El modelo ha servido para poder distinguir entre los dos factores cuantitativos y demográficos específicos que se estiman en un indicador de fecundidad que condicionan el resultado. Así, se ha separado el denominado efecto estructura de una diferencia en los niveles que se reconoce como perdurable. Este ejercicio ficticio apunta a demostrar:

1. Que siempre hay y habrá diferencias.

2. Que no tiene sentido buscar la igualdad total o la homogeneización de poblaciones tan distintas como la de un país o la proveniente de migraciones recientes.

3. Que, al contrario de lo que muchas veces se estima, las diferencias no son ni tan grandes ni tan irreconciliables. El hecho de que la tendencia de los indicadores fuera la inversa, apuntaría a una cierta convergencia, aunque nunca a un punto de encuentro que las iguale. Esta circunstancia quedó demostrada con la inflexión de los últimos años.

4. Todavía queda mucho camino por recorrer. Pasará tiempo antes de que la fecundidad de los diferentes orígenes se aproxime aún más. También cambiarán las cosas por otra razón: si las hijas han nacido en España, las diferencias puede que sean naturalmente menores.

Todo está por ver, pero ello no es motivo de alarma.

\section{Referencias bibliográficas}

ANDORKA, Rudolf (1982). Determinants of fertility in advanced societies. Londres: Methuen and Co.

BONGAARTS, John y COTTS WATKINS, Susan (1996). «Social interaction and contemporary fertility transitions». Population and Development Review, 22, 4.

De VAlK, H.; LiefBroer, A.C.; EsveldT, I. y HenKENS. K(2004). «Family formation and cultural integration among migrants in the Netherlands». Genus, LX, 3-4, 9-35.

DURKHEIM, Emile (1895). Les règles de la méthode sociologique. París: PUF, 1959. 
INE (2008). Nacimientos y poblaciones de referencia. Madrid: Instituto Nacional de Estadística de España. [www.ine.es, consulta: junio de 2008, actualización: febrero de 2010]

SARRIBLE, Graciela (1986). Estudio de la fecundidad de la población nativa y migrante de la ciudad de Barcelona. Tesis doctoral defendida en la Universidad Complutense de Madrid.

- (2004). «Fertility of foreing migrant women in Spain». International Joint Congress, European Society for Health and Medical Sociology. Bolonia (Italia).

- (2005). "L'apport des étrangères à l'acrossement de la population en Espagne». Migrations et sociétés. Dossier: De l'èmigration à l'inmigration en Europe et ailleurs, XVII, $102,293-303$.

- (2006). «Embarazos de adolescentes extranjeras en España: ser madre o abortar». Comunicación presentada en la European Association of Global Bioethics International Congress on Multiculturalism and Health. Barcelona, marzo. Publicado en la Revista de Bioética y Derecho, 8.

- (2008). «Españolas y extranjeras en España: desigualdades en cuestiones de fecundidad, 1999-2004». Revista de Bioética y Derecho, 13, 44-49. [www.ub.es/fildt/revista7index.htm] 